\title{
粒子法における時間進行法の改良
}

松永 拓也 ${ }^{* 1}$, 越塚 誠一 ${ }^{* 2}$

\section{Improvement of the time marching method in a particle method}

\author{
Takuya MATSUNAGA ${ }^{* 1}$ and Seiichi KOSHIZUKA ${ }^{* 2}$ \\ ${ }^{*} 1,{ }^{* 2}$ Department of Systems Innovation, The University of Tokyo \\ 7-3-1 Hongo, Bunkyo-ku, Tokyo 113-8656, Japan
}

\section{Received: 9 December 2020; Revised: 17 February 2021; Accepted: 28 February 2021}

\begin{abstract}
This study concerns the computational accuracy of a particle method for a time-dependent incompressible flow. In recent years, accurate spatial discretization schemes have been developed for a particle method. However, the actual convergence rate in space tends to be much lower than the order of the leading truncation error given by an adopted spatial discretization scheme. This suggests that the $\Delta t$-dependent error is comparably significant with respect to the truncation error of the spatial discretization scheme. Under these circumstances, we have developed a new time marching method to improve the computational accuracy by reducing the $\Delta t$-dependent error and improving the convergence property. The proposed time marching method is based on the 1st-order fractional step method, just as the conventional methods. However, as opposed to the past studies, the convection term is explicitly included in the provisional velocity calculation, as an Eulerian-based approach. By doing this, the $\Delta t$-dependent error caused by the particle movement can be avoided. A numerical test has been carried out using the two-dimensional Taylor-Green vortex problem, where 2nd- and 4thorder spatial discretization schemes are adopted. As a result, the conventional time marching methods gave much lower convergence rate than the order of the spatial discretization scheme. On the other hand, the proposed time marching method showed approximately 2nd- and 4th-order convergence in space with the 2nd- and 4th-order spatial discretization schemes, respectively. Therefore, the proposed method is indicated to highly improve the computational accuracy.
\end{abstract}

Keywords : Computational fluid dynamics, Incompressible flow, Arbitrary Eulerian Lagrangian method, Fractional step method, LSMPS method

\section{1. 緒言}

粒子法は複雑な自由表面流れを数值シミュレーションできることから機械工学やコンピュータグラフィックス 等の分野で多く利用されている。広く普及した手法として, SPH (smoothed particle hydrodynamics) 法 (Monaghan, 1992) と MPS (moving particle semi-implicit) 法 (Koshizuka and Oka, 1996) が挙げられる.これまでにダム崩壊, 砕 波，スロッシング，津波，擋汼，二軸押出機など工学分野で現れる様々な問題に適用されており，産業の場でも実 用化さ水始めている (Violeau and Rogers, 2016; Gotoh and Khayyer, 2016; Koshizuka et al., 2018). しかし，計算精 度に課題があり, 厳密さが求められる解析には適さないという指摘がある.

計算精度を改善した粒子法に LSMPS (least squares moving particle semi-implicit) 法 (Tamai and Koshizuka, 2014)

\footnotetext{
No.20-00437 [DOI:10.1299/transjsme.20-00437], J-STAGE Advance Publication date : 9 March, 2021

*1 正員, 東京大学大学院工学系研究科 システム創成学専攻（广113-8656 東京都文京区本郷 7-3-1）

*2 正員, フェロー, 東京大学大学院工学系研究科システム創成学専攻

E-mail of corresponding author: matsunaga@mps.q.t.u-tokyo.ac.jp
} 
がある．従来手法との最も大きな違いは，空間離散化スキームを高精度化したことである．LSMPS 法では空間微 分の離散化に LSMPS スキームを用いる. LSMPS スキームは Taylor 展開と最小二乗法に基づいて定式化されてお り，理論上は支配方程式に含まれる全ての空間微分を任意次数の精度で計算できる。しかし，粒子分布の偏りな どに起因して数值不安定化しやすい傾向があり, 安定性の改善が課題であった.

最近の研究では, 粒子の移動を完全に流れに追従させる Lagrange 法でなく, 任意の粒子移動を扱える ALE (arbitrary Lagrangian-Eulerian) 法を採用する動きがある (Xu et al., 2009; Oger et al., 2016; Khayyer et al., 2017; Duan et al., 2018). Lagrange 法と比較したときの ALE 法の利点は, 物理的な流体速度とは独立に粒子の移動を決定で きるため, 計算精度を大きく損なうことなく粒子分布を均一化するような補正を加えられる点にある．従って, Lagrange 法では時間進行に伴い粒子の粗密が蓄積して数值不安定化が起こるのに対し, ALE 法では常におおよそ 均一な粒子分布が得られるため安定性が飛躍的に向上する。

既に LSMPS 法に対してALE の定式化を適用した手法が開発されており, 数值安定性が改善されている(Hu et al., 2017; Matsunaga et al., 2020; Wang et al., 2020). 従って, 2 次精度あるいは 4 次精度の LSMPS スキームを用い ることにより, 空間 2 次や 4 次の収束性を有する高精度な数值解析が期待される。 ところが, ベンチマーク問題 を用いて実際に数值誤差を評価してみると, 非定常解析における瞬時速度場の数值解は期待されるよりずっと低 いオーダーでしか収束しない。これには空間離散化とは独立に発生する時間ステップ $(\Delta t)$ 依存の誤差が大きく影 響している。

粒子法では一般に部分段階法に基づく時間進行法が用いられる. 速度の時間微分を Euler 陽解法 (あるいは Euler 陰解法) で離散化するため, 時間 1 次精度の手法であると言える. 同様の時間進行法を有限差分法などの Euler 法 に適用した場合にも, 計算点の移動の有無に違いがあるのみで, 理論上は同じく時間 1 次精度である.ところが, Euler 法に対して数值誤差の収束性を評価してみると, 速度場の誤差ノルムが空間離散化の打ち切り誤差に準ずる オーダーで減少することがしばしばある。これは $\Delta t$ 依存の誤差に比べて空間離散化の誤差が支配的であることを 意味する。つまり粒子法では対照的に， $\Delta t$ 依存の誤差が支配的，あるいは少なくとも空間離散化の誤差に匹敵す る大きさで発生していると考えられる。そのため, 高次の空間離散化スキームを使用したとしても, 実質的な数 值䛊差の収束速度は $\Delta t$ に支配されてしまい，高精度スキームの恩恵を受けることができない.

そこで本研究は, 粒子法において収束を阻害する要因になっていると考元られる $\Delta t$ 依存の誤差の原因を突き止 め, 定式化ならびに時間進行法の改良によってその誤差項の発生を根本的に回避し, 計算精度を向上することを 目的とする. 本稿の構成としては，2 章にて前提とする支配方程式と空間離散化スキームについて述べる. 3 章で 従来手法の問題点を指摘し，4章で提案手法を説明する．5 章では二次元 Taylor-Green 渦を用いて解の収束性を検 証し，提案手法によって計算精度が向上することを示す。

\section{2. 支配方程式と空間離散化}

\section{$2 \cdot 1$ 支配方程式}

密度および粘度一定のニュートン流体の非圧縮性流れを扱うものとし, 以下に示す連続の式と Navier-Stokes 方 程式を支配方程式とする。

$$
\nabla \cdot \boldsymbol{u}=0
$$

$$
\frac{\partial \boldsymbol{u}}{\partial t}+(\boldsymbol{u} \cdot \nabla) \boldsymbol{u}=-\frac{1}{\rho} \nabla P+v \nabla^{2} \boldsymbol{u}+\boldsymbol{g}
$$

ここで $t$ は時間， $u$ は速度， $P$ は圧力， $\rho$ は密度， $v$ は動粘性係数， $g$ は外力を表す． 


\section{$2 \cdot 2$ 空間離散化}

空間微分の離散化には LSMPS スキーム (Tamai and Koshizuka, 2014) を用いる. 関数 $\phi$ の位置 $\boldsymbol{x}_{i}$ における空間 微分は LSMPS スキームを用いて次のように離散化される。

$$
\begin{aligned}
& \left.\boldsymbol{D} \phi\right|_{\boldsymbol{x}_{i}}=H_{r_{\mathrm{s}}} M_{i}^{-1} \sum_{j} w_{i j} \boldsymbol{p}_{i j}\left(\phi_{j}-\phi_{i}\right) \\
& M_{i}=\sum_{j} w_{i j} \boldsymbol{p}_{i j} \otimes \boldsymbol{p}_{i j}
\end{aligned}
$$

ここで $w_{i j}$ は重み関数, $\boldsymbol{p}_{i j}$ は多項式基底ベクトル， $H_{r_{\mathrm{s}}}$ はスケーリング行列， $M_{i}$ はモーメント行列, $\boldsymbol{D}$ は微分作 用素ベクトルを表す。 $p$ 次の LSMPS スキームについて, 自然数の組から成るマルチインデックス $\boldsymbol{\alpha}=(\alpha, \beta, \gamma)$ を 用いて成分表示すると以下のようになる。

$$
\begin{aligned}
& \boldsymbol{p}_{i j}=\boldsymbol{p}\left(\frac{\boldsymbol{x}_{j}-\boldsymbol{x}_{i}}{r_{\mathrm{s}}}\right)=\left[\frac{\left(\boldsymbol{x}_{j}-\boldsymbol{x}_{i}\right)^{\boldsymbol{\alpha}}}{r_{\mathrm{s}}^{|\boldsymbol{\alpha}|}}\right]_{1 \leq|\boldsymbol{\alpha}| \leq p}=\left[\frac{\left(x_{j}-x_{i}\right)^{\alpha}\left(y_{j}-y_{i}\right)^{\beta}\left(z_{j}-z_{i}\right)^{\gamma}}{r_{\mathrm{s}}^{\alpha+\beta+\gamma}}\right]_{1 \leq \alpha+\beta+\gamma \leq p} \\
& H_{r_{\mathrm{s}}}=\operatorname{diag}\left(\left[\frac{\boldsymbol{\alpha} !}{r_{\mathrm{s}}^{|\boldsymbol{\alpha}|}}\right]_{1 \leq|\boldsymbol{\alpha}| \leq p}\right)=\operatorname{diag}\left(\left[\frac{\alpha ! \beta ! \gamma !}{r_{\mathrm{s}}^{\alpha+\beta+\gamma}}\right]_{1 \leq \alpha+\beta+\gamma \leq p}\right) \\
& \boldsymbol{D}=\left[D_{\boldsymbol{x}}^{\boldsymbol{\alpha}}\right]_{1 \leq|\boldsymbol{\alpha}| \leq p}=\left[\frac{\partial^{\alpha+\beta+\gamma}}{\partial x^{\alpha} \partial y^{\beta} \partial z^{\gamma}}\right]_{1 \leq \alpha+\beta+\gamma \leq p}
\end{aligned}
$$

ここで $r_{\mathrm{s}}$ はスケーリング係数である. スケーリング係数 $r_{\mathrm{s}}$ は空間次元数 $d$, 流体体積 $|\Omega|$, 粒子数 $N$ で定義され る次の代表粒子間距離 $\Delta x$ の定数倍とする.

$$
\Delta x=\left(\frac{|\Omega|}{N}\right)^{1 / d}
$$

また，重み関数は次のように定義する.

$$
\begin{aligned}
& w_{i j}=w\left(\left\|\boldsymbol{x}_{j}-\boldsymbol{x}_{i}\right\|\right) \\
& w(r)= \begin{cases}\left(1-\frac{r}{r_{\mathrm{e}}}\right)^{2} & \left(r<r_{\mathrm{e}}\right) \\
0 & \left(r \geq r_{\mathrm{e}}\right)\end{cases}
\end{aligned}
$$

ここで $r_{\mathrm{e}}$ は影響半径である.

\section{3. 既存の時間進行法}

\subsection{Euler 法の場合}

有限差分法や有限体積法は Euler 法を用いた計算手法である. Euler 法は計算点の移動を考慮しないため, 以下 のような単純な部分段階法によって時間進行を計算できる.

$$
\frac{\boldsymbol{u}^{*}-\boldsymbol{u}^{k}}{\Delta t}=-(\boldsymbol{u} \cdot \nabla) \boldsymbol{u}+v \nabla^{2} \boldsymbol{u}+\boldsymbol{g}
$$




$$
\frac{\boldsymbol{u}^{k+1}-\boldsymbol{u}^{*}}{\Delta t}=-\frac{1}{\rho} \nabla P
$$

ここで定義される一時変数 $\boldsymbol{u}^{*}$ を仮速度と呼ぶ. 圧力 $P$ は次の圧力ポアソン方程式を解いて求められる.

$$
\nabla \cdot\left(\frac{1}{\rho} \nabla P\right)=\frac{\nabla \cdot \boldsymbol{u}^{*}}{\Delta t}
$$

この時間進行法は速度を時間方向に Taylor 展開した

$$
\boldsymbol{u}(\boldsymbol{x}, t+\Delta t)=\boldsymbol{u}(\boldsymbol{x}, t)+\Delta t \frac{\partial \boldsymbol{u}}{\partial t}+\frac{\Delta t^{2}}{2 !} \frac{\partial^{2} \boldsymbol{u}}{\partial t^{2}}+\frac{\Delta t^{3}}{3 !} \frac{\partial^{3} \boldsymbol{u}}{\partial t^{3}}+\cdots
$$

を一次の項で打ち切ったものに対応する. 従って, 空間離散化に伴う打ち切り誤差を $\mathscr{O}\left(\Delta x^{p}\right)$ とおくと, 各時間ス テップの速度増分に含まれる誤差は

$$
\boldsymbol{u}^{k+1}-\boldsymbol{u}^{k}=\Delta t \frac{\partial \boldsymbol{u}}{\partial t} \approx \Delta t\left(\frac{\partial \boldsymbol{u}}{\partial t}+\mathscr{O}\left(\Delta x^{p}\right)\right)+\mathscr{O}\left(\Delta t^{2}\right)
$$

である. $\mathscr{O}\left(\Delta x^{p}\right)$ と $\mathscr{O}\left(\Delta t^{2}\right)$ のどちらか一方が極めて小さければ, $K$ ステップ後の蓄積誤差は

$$
\frac{\boldsymbol{u}^{K}-\boldsymbol{u}_{\mathrm{exact}}^{K}}{t_{K}}=\mathscr{O}\left(\Delta x^{p}\right)+\mathscr{O}(\Delta t)
$$

と見積もられる。よって, 非定常解析における瞬時速度場の数值誤差は空間 $p$ 次・時間 1 次で収束することを表 す．従って，十分に小さな $\Delta t$ のもとで空間方向 $(\Delta x \rightarrow 0)$ の収束性を評価すれば，速度場の誤差ノルムは $p$ 次の オーダーで減少することが期待される。ただし， $\Delta t$ をどの程度小さくとれば十分かは対象とする問題や解法に依 存する.もし数值安定性の条件から与えられる $\Delta t$ の上限值付近で上記要件を満たすなら, ょり高次の適合性を有 する空間離散化スキームを用いることで効果的な精度向上が望める。一方で, $\Delta t$ 依存の誤差が $\Delta x$ 依存の誤差に 匹敵する割合で存在している場合には， $\Delta t \propto \Delta x^{1}$ のもとで収束をとると次第に $\mathscr{O}(\Delta t)$ が支配的となる．このとき， 高次の適合性を有する空間離散化スキームを用いてもほとんど意味をなさない.

以上のことを念頭に置いた上で，粒子法における従来の時間進行法を振り返ることとする.

\subsection{Koshizuka and Oka の方法}

Koshizuka and Oka (1996)の MPS 法で採用される時間進行法について説明する。なお，ここでは時間進行法と 対流項の取り扱いに着目することとし，議論の本筋から外音部分に関しては必ずしも原著論文に従わないこと に注意されたい.

Koshizuka and Oka の方法は部分段階法に基づいており，以下の手順で時間進行を計算する.

(1) 仮速度 $\boldsymbol{u}^{*}$ の計算を行う。

$$
\boldsymbol{u}^{*}=\boldsymbol{u}^{k}+\Delta t\left[v \nabla^{2} \boldsymbol{u}^{k}+\boldsymbol{g}\right]
$$

（2）粒子を仮位置 $\boldsymbol{r}^{*}$ に移動する.

$$
\boldsymbol{r}^{*}=\boldsymbol{r}^{k}+\Delta t \boldsymbol{u}^{*}
$$


(3) 圧力ポアソン方程式を解いて圧力 $P^{k+1}$ を求める.

$$
\nabla^{2} P^{k+1}=\frac{\rho}{\Delta t} \nabla \cdot \boldsymbol{u}^{*}
$$

(4) 速度と位置を修正して新しい時刻の值を得る.

$$
\begin{aligned}
& \boldsymbol{u}^{k+1}=\boldsymbol{u}^{*}-\frac{\Delta t}{\rho} \nabla P^{k+1} \\
& \boldsymbol{r}^{k+1}=\boldsymbol{r}^{*}-\frac{\Delta t^{2}}{\rho} \nabla P^{k+1}
\end{aligned}
$$

この時間進行法にはいくつか問題点がある。一つは対流項の考慮方法にある. Lagrange 法では計算点の移動に よって対流項を考慮するため，対流項を陽的に計算式に含める必要がないと考えられている．Koshizuka and Oka の方法もその考え方に従っており，対流項は現れない。より詳しく説明すると，ある時刻における近傍の二点間の 速度差は Taylor 展開を用いて

$$
\boldsymbol{u}\left(\boldsymbol{r}^{\prime}, t\right)-\boldsymbol{u}(\boldsymbol{r}, t)=\left.\sum_{m=1}^{\infty} \frac{1}{m !}\left(\left(\boldsymbol{r}^{\prime}-\boldsymbol{r}\right) \cdot \nabla\right)^{m} \boldsymbol{u}\right|_{\boldsymbol{r}} ^{t}
$$

と記述できる。従って，仮位置 $\boldsymbol{r}^{*}$ に定義された仮速度 $\boldsymbol{u}^{*}$ は次のような近似を表す。

$$
\begin{aligned}
\boldsymbol{u}^{*} & =\boldsymbol{u}^{k}+\Delta t\left[v \nabla^{2} \boldsymbol{u}^{k}+\boldsymbol{g}\right] \\
& \approx \boldsymbol{u}\left(\boldsymbol{r}^{*}, t_{k}\right)-\left.\sum_{m=1}^{\infty} \frac{1}{m !}\left(\Delta t \boldsymbol{u}^{*} \cdot \nabla\right)^{m} \boldsymbol{u}\right|_{\boldsymbol{r}^{k}} ^{t_{k}}+\Delta t\left[\left.v \nabla^{2} \boldsymbol{u}\right|_{\boldsymbol{r}^{k}} ^{t_{k}}+\boldsymbol{g}\right] \\
& =\boldsymbol{u}\left(\boldsymbol{r}^{*}, t_{k}\right)+\Delta t\left[-\left.\left(\boldsymbol{u}^{*} \cdot \nabla\right) \boldsymbol{u}\right|_{\boldsymbol{r}^{k}} ^{t_{k}}+\left.v \nabla^{2} \boldsymbol{u}\right|_{\boldsymbol{r}^{k}} ^{t_{k}}+\boldsymbol{g}\right]-\left.\sum_{m=2}^{\infty} \frac{\Delta t^{m}}{m !}\left(\boldsymbol{u}^{*} \cdot \nabla\right)^{m} \boldsymbol{u}\right|_{\boldsymbol{r}^{k}} ^{t_{k}}
\end{aligned}
$$

確かに，対流項に類似するものが考虑されていることがわかる。しかし， $\Delta t^{2}$ 以上の項が含まれることに加え， $\left(\boldsymbol{u}^{*} \cdot \nabla\right) \boldsymbol{u} \neq(\boldsymbol{u} \cdot \nabla) \boldsymbol{u}$ であるから，式 (11) で与えられる仮速度の定義には一致しない. ただし，式 (21)によって粒 子位置は更に $\boldsymbol{r}^{*}$ から $\boldsymbol{r}^{k+1}$ に移動するから，新しい時刻の速度 $\boldsymbol{u}^{k+1}$ は

$$
\boldsymbol{u}^{k+1} \approx \boldsymbol{u}\left(\boldsymbol{r}^{k+1}, t_{k}\right)+\Delta t\left[-\left.\left(\boldsymbol{u}^{k+1} \cdot \nabla\right) \boldsymbol{u}\right|_{\boldsymbol{r}^{k+1}} ^{t_{k}}+\left.v \nabla^{2} \boldsymbol{u}\right|_{\boldsymbol{r}^{k}} ^{t_{k}}+\boldsymbol{g}-\frac{1}{\rho} \nabla P\right]-\left.\sum_{m=2}^{\infty} \frac{\Delta t^{m}}{m !}\left(\boldsymbol{u}^{k+1} \cdot \nabla\right)^{m} \boldsymbol{u}\right|_{\boldsymbol{r}^{k+1}} ^{t_{k}}
$$

となって，最終的には $\boldsymbol{u}^{k+1}$ を用いて評価された対流項として正しく修正されると主張することもできる，ところ が，圧力ポアソン方程式には仮速度の発散が含まれているため，仮速度に含まれる対流項の誤差は圧力の誤差と なって現れる。 また，仮に式(18)を

$$
\boldsymbol{r}_{i}^{*}=\boldsymbol{r}_{i}^{k}+\Delta t \boldsymbol{u}_{i}^{k}
$$

に置き換えたとしても，仮速度が実際に計算された位置 $\left(\boldsymbol{r}^{k}\right)$ と圧力ポアソン方程式中で定義される位置 $\left(\boldsymbol{r}^{*}\right)$ にず れがあるため, やはり本来の圧力ポアソン方程式には一致しない. 要約すると, $\Delta t$ 依存の誤差が粒子移動に伴っ て発生しているのである. 
結局，Koshizuka and Oka の方法では各時間ステップの速度増分に以下のような誤差が現れる.

$$
\boldsymbol{u}^{k+1}-\boldsymbol{u}^{k} \approx \Delta t\left(\frac{\partial \boldsymbol{u}}{\partial t}+\mathscr{O}\left(\Delta x^{p}\right)+\mathscr{O}(\Delta t)\right)+\mathscr{O}\left(\Delta t^{2}\right)
$$

オーダーのみに着目すると Euler 法の部分段階法と同じであるようにも思えるが，粒子移動に伴う $\mathscr{O}(\Delta t)$ の䛊差が $\mathscr{O}\left(\Delta x^{p}\right)$ と同程度あるいはそれ以上の大きさを有するため，実際には全く異なる収束特性を示すこととなる．当然 ながら， $\Delta t$ に依存する誤差は $\Delta t \rightarrow 0$ の極限で消えることから，収束解には影響しないと考えることもできる．し かし，解の精度と収束性を悪くすることは明白であり，除去することが望ましい.

\subsection{Cummins and Rudman の方法}

Cummins and Rudman (1999)の SPH 法では，以下のように時間進行する.

(1) 粒子を仮位置 $\boldsymbol{r}^{*}$ に移動する.

$$
\boldsymbol{r}^{*}=\boldsymbol{r}^{k}+\Delta t \boldsymbol{u}^{k}
$$

（2）仮速度 $\boldsymbol{u}^{*}$ を計算する.

$$
\boldsymbol{u}^{*}=\boldsymbol{u}^{k}+\Delta t\left[v \nabla^{2} \boldsymbol{u}^{k}+\boldsymbol{g}\right]
$$

(3) 圧力ポアソン方程式を解いて圧力を求める.

$$
\nabla^{2} P^{k+1}=\frac{\rho}{\Delta t} \nabla \cdot u^{*}
$$

(4) 速度と位置を修正する.

$$
\begin{aligned}
& \boldsymbol{u}^{k+1}=\boldsymbol{u}^{*}-\frac{\Delta t}{\rho} \nabla P^{k+1} \\
& \boldsymbol{r}^{k+1}=\boldsymbol{r}^{k}+\Delta t\left(\frac{\boldsymbol{u}^{k}+\boldsymbol{u}^{k+1}}{2}\right)
\end{aligned}
$$

Cummins and Rudman の方法も Koshizuka and Oka の方法と同様に Lagrange 法を採用しており，計算式に対流 項は含まれない. しかし, 式 (29) に示されるように, 仮速度 $\boldsymbol{u}^{*}$ でなく, 古い時刻の速度 $\boldsymbol{u}^{k}$ で粒子を移動する. そ れによって，仮位置 $\boldsymbol{r}^{*}$ に定義された速度 $\boldsymbol{u}^{k}$ は

$$
\boldsymbol{u}^{k} \approx \boldsymbol{u}\left(\boldsymbol{r}^{*}, t_{k}\right)+\Delta t\left[-\left.\left(\boldsymbol{u}^{k} \cdot \nabla\right) \boldsymbol{u}\right|_{\boldsymbol{r}^{*}} ^{t_{k}}\right]+\left.\sum_{m=2}^{\infty} \frac{\Delta t^{m}}{m !}\left(-\boldsymbol{u}^{k} \cdot \nabla\right)^{m} \boldsymbol{u}\right|_{\boldsymbol{r}^{*}} ^{t_{k}}
$$

となるため, 対流項の計算精度が改善されている，ところが, 式(30)の仮速度の計算では, この対流項を含んだ 速度を用いて粘性項を評価しており，

$$
\nabla^{2} \boldsymbol{u}^{k} \approx \nabla^{2}\left[\boldsymbol{u}+\sum_{m=1}^{\infty} \frac{\Delta t^{m}}{m !}\left(-\boldsymbol{u}^{k} \cdot \nabla\right)^{m} \boldsymbol{u}\right]_{\boldsymbol{r}^{*}}^{t_{k}} \neq\left.\nabla^{2} \boldsymbol{u}\right|_{\boldsymbol{r}^{*}} ^{t_{k}}
$$

となることから粘性項に不適合性が生じる，従って，仮速度に $\Delta t$ 依存の誤差を有しており，Koshizuka and Okaの 方法と同様の問題点がある. 


\subsection{Matsunaga et al. の方法}

Matsunaga et al. (2020) の解法はALE 法を採用しており, 粒子分布の均一化補正を粒子移動に加えている. 具体 的な計算手順は以下の通りである.

(1) 仮速度 $\boldsymbol{u}^{*}$ を計算する。

$$
\boldsymbol{u}^{*}=\boldsymbol{u}^{k}+\delta \boldsymbol{u}^{k}+\Delta t\left[v \nabla^{2} \boldsymbol{u}^{k}+\boldsymbol{g}\right]
$$

ここで $\delta \boldsymbol{u}^{k}$ は ALE 化に伴う対流項の補正を表しており，次のように定義される.

$$
\left.\delta \boldsymbol{u}^{k} \approx \sum_{m=1}^{\infty} \frac{1}{m !}\left(\delta \boldsymbol{r}^{k} \cdot \nabla\right)^{m} \boldsymbol{u}\right|_{\boldsymbol{r}^{k}} ^{t_{k}}
$$

ここで $\delta \boldsymbol{r}^{k}$ は粒子位置の補正である。 また， $\delta \boldsymbol{u}^{k}$ は実際には $p$ 次の項で打ち切った式を用いて計算する。

(2) 粒子を位置 $\boldsymbol{r}^{k+1}$ に移動する.

$$
\boldsymbol{r}^{k+1}=\boldsymbol{r}^{k}+\Delta t \boldsymbol{u}^{k}+\delta \boldsymbol{r}^{k}
$$

(3) 圧力ポアソン方程式を解いて圧力を求める.

$$
\nabla^{2} P^{k+1}=\frac{\rho}{\Delta t} \nabla \cdot \boldsymbol{u}^{*}
$$

(4) 速度を修正する.

$$
\boldsymbol{u}^{k+1}=\boldsymbol{u}^{*}-\frac{\Delta t}{\rho} \nabla P^{k+1}
$$

位置 $\boldsymbol{r}^{k+1}$ に定義される仮速度 $\boldsymbol{u}^{*}$ に着目すると, 位置補正 $\delta \boldsymbol{r}^{k}$ による速度の変化分と $\delta \boldsymbol{u}^{k}$ とが打ち消し合うため,

$$
\boldsymbol{u}^{*} \approx \boldsymbol{u}\left(\boldsymbol{r}^{*}, t_{k}\right)+\Delta t\left[-\left.\left(\boldsymbol{u}^{k} \cdot \nabla\right) \boldsymbol{u}\right|_{\boldsymbol{r}^{k}} ^{t_{k}}+\left.v \nabla^{2} \boldsymbol{u}\right|_{\boldsymbol{r}^{k}} ^{t_{k}}+\boldsymbol{g}\right]-\left.\sum_{m=2}^{\infty} \frac{\Delta t^{m}}{m !}\left(\boldsymbol{u}^{k} \cdot \nabla\right)^{m} \boldsymbol{u}\right|_{\boldsymbol{r}^{k}} ^{t_{k}}
$$

となる。これは Koshizuka and Oka の方法において，仮位置の計算を式 (27) に置き換えた場合の結果に一致する. 従って, Koshizuka and Oka の方法に比べて対流項が改善されており，また，Cummins and Rudmanの方法に比べ て粘性項が改善されている。しかし, 大きく二つの問題点が残っている.

一つは, Lagrange 法に基づく対流項にある. 式 (41) に示されるように， $\Delta t^{2}$ 以上の高次の項が粒子移動によっ て発生している。これらの項は速度の実質微分に高次の項を加えたもの，あるいは対流項の時間積分を考えたも のとして解釈することもできる。しかし，これらは本来，式 (14)における $\partial^{2} \boldsymbol{u} / \partial t^{2} や \partial^{3} \boldsymbol{u} / \partial t^{3}$ 等に現れるもので あって，いま想定している $\partial u / \partial t$ ベースの部分段階法には不適であると考える。つまり，ここで考慮される対流 項には $\mathscr{O}\left(\Delta t^{2}\right)$ の誤差が含まれていると見做すべきである.

もう一つの問題点は，圧力ポアソン方程式における仮速度の誤差である. Koshizuka and Oka の方法と同様に， 仮速度の速度増分 (つまり対流項と粘性項) が評価された位置 $\left(\boldsymbol{r}^{k}\right)$ と圧力ポアソン方程式で発散を評価するときに 定義されている位置 $\left(\boldsymbol{r}^{k+1}\right)$ が一致していない。これに伴い, 圧力に $\left\|\boldsymbol{r}^{k+1}-\boldsymbol{r}^{k}\right\|$ 依存の誤差が発生する.

\section{4. 提案の時間進行法}

ここで提案する時間進行法はALE 法を対象としている。従って，任意の粒子移動を扱うことができるため， Lagrange 法や Euler 法に対しても適用できる。具体的な計算手順を以下に示す. 
（1）仮速度 $\boldsymbol{u}^{*}$ を計算する。

$$
\boldsymbol{u}^{*}=\boldsymbol{u}^{k}+\Delta t\left[-\left(\boldsymbol{u}^{k} \cdot \nabla\right) \boldsymbol{u}^{k}+v \nabla^{2} \boldsymbol{u}^{k}+\boldsymbol{g}\right]
$$

(2) 圧力ポアソン方程式を解いて圧力を求める.

$$
\nabla^{2} P^{k}=\frac{\rho}{\Delta t} \nabla \cdot \boldsymbol{u}^{*}
$$

(3) 新しい時刻の速度 $\tilde{\boldsymbol{u}}^{k+1}$ を計算する.

$$
\tilde{\boldsymbol{u}}^{k+1}=\boldsymbol{u}^{*}-\frac{\Delta t}{\rho} \nabla P^{k}
$$

（4）粒子移動による速度補正を計算する.

$$
\boldsymbol{u}^{k+1}=\tilde{\boldsymbol{u}}^{k+1}+\sum_{m=1}^{p} \frac{1}{m !}\left(\Delta \boldsymbol{r}^{k} \cdot \nabla\right)^{m} \tilde{\boldsymbol{u}}^{t_{k+1}}
$$

(5) 粒子を移動する.

$$
\boldsymbol{r}^{k+1}=\boldsymbol{r}^{k}+\Delta \boldsymbol{r}^{k}
$$

提案手法の特徵は，対流項と粒子移動による速度変化を分離して考慮していることである．式 (42)では陽的に 対流項を計算式に組み込み, 式 (45) では粒子移動による速度変化を Taylor 展開近似により求めている. 最終的に 位置 $\boldsymbol{r}^{k+1}$ に定義される新しい速度 $\boldsymbol{u}^{k+1}$ は

$$
\begin{aligned}
\boldsymbol{u}^{k+1} & =\boldsymbol{u}^{k}+\Delta t\left[-\frac{1}{\rho} \nabla P^{k}-\left(\boldsymbol{u}^{k} \cdot \nabla\right) \boldsymbol{u}^{k}+v \nabla^{2} \boldsymbol{u}^{k}+\boldsymbol{g}\right]+\sum_{m=1}^{p} \frac{1}{m !}\left(\Delta \boldsymbol{r}^{k} \cdot \nabla\right)^{m} \tilde{\boldsymbol{u}}^{t_{k+1}} \\
& =\sum_{m=0}^{p} \frac{1}{m !}\left(\Delta \boldsymbol{r}^{k} \cdot \nabla\right)^{m}\left(\boldsymbol{u}^{k}+\Delta t\left[-\frac{1}{\rho} \nabla P^{k}-\left(\boldsymbol{u}^{k} \cdot \nabla\right) \boldsymbol{u}^{k}+v \nabla^{2} \boldsymbol{u}^{k}+\boldsymbol{g}\right]\right) \\
& \approx \boldsymbol{u}\left(\boldsymbol{r}^{k+1}, t_{k}\right)+\Delta t\left[-\frac{1}{\rho} \nabla P-\left(\boldsymbol{u}^{k} \cdot \nabla\right) \boldsymbol{u}+v \nabla^{2} \boldsymbol{u}+\boldsymbol{g}\right]_{\boldsymbol{r}^{k+1}}^{t_{k}}+\mathscr{O}\left(\left\|\Delta \boldsymbol{r}^{k}\right\|^{p+1}\right)
\end{aligned}
$$

の近似を与えるものとなっており，対流項の評価に伴う $\Delta t$ 依存の項が発生しないことがわかる．また，仮速度が 計算されてから圧力ポアソン方程式を解くまでの間に粒子の移動がないため，仮速度の定義位置のずれの問題が 解消されている。従って, 従来手法において精度低下の要因として指摘された粒子移動に伴う $\Delta t$ 依存の誤差の発 生が提案手法では根本的に回避されており, Euler 法の部分段階法と同様の収束特性を得られることが期待される. なお，上記の方法では粘性項を陽的に扱っているが，陰的に計算する場合は式 (44)を

$$
\tilde{\boldsymbol{u}}^{k+1}-\theta \Delta t v \nabla^{2} \tilde{\boldsymbol{u}}^{k+1}=\boldsymbol{u}^{*}-\theta \Delta t v \nabla^{2} \boldsymbol{u}^{k}-\frac{\Delta t}{\rho} \nabla P^{k}
$$

に置き換えればよい，これは，次式のように粘性項に対して $\theta$ 法を適用したことに相当する.

$$
\frac{\tilde{\boldsymbol{u}}^{k+1}-\boldsymbol{u}^{k}}{\Delta t}=-\frac{1}{\rho} \nabla P^{k}-\left(\boldsymbol{u}^{k} \cdot \nabla\right) \boldsymbol{u}^{k}+(1-\theta) v \nabla^{2} \boldsymbol{u}^{k}+\theta v \nabla^{2} \tilde{\boldsymbol{u}}^{k+1}+\boldsymbol{g} \quad(0 \leq \theta \leq 1)
$$




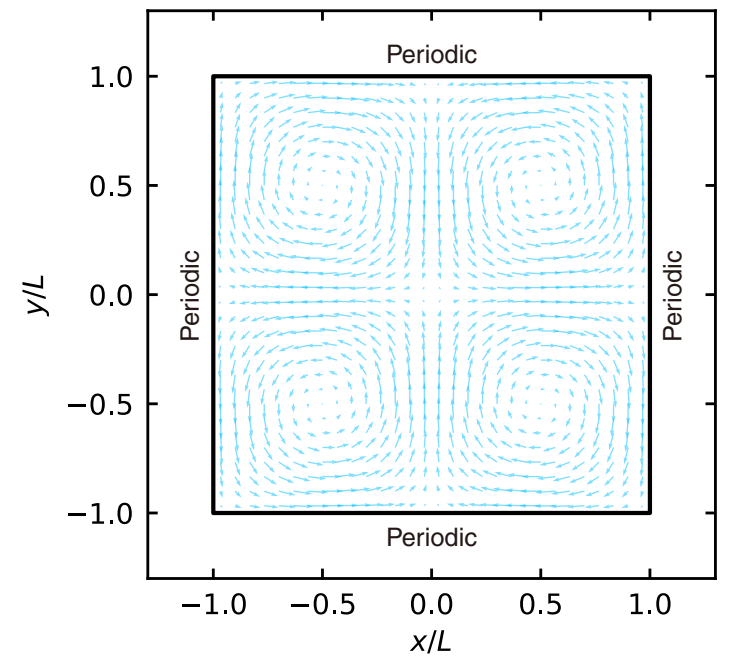

Fig. 1 Computational domain of the Taylor-Green vortex problem. Arrows indicate initial velocity vectors.

提案手法における圧力の定義について補足すると, 従来の $P^{k+1}$ から $P^{k}$ に置き換わつており, 圧力ポアソン方 程式を解いて求められる圧力值は新しい時刻 $t_{k+1}$ でなく古い時刻 $t_{k}$ で定義されるものである. これは, 時刻 $t_{k} に$ おける Navier-Stokes 方程式の発散をとって得られる

$$
\nabla \cdot\left(\frac{1}{\rho} \nabla P^{k}\right)=\nabla \cdot\left(-\left.\frac{\partial \boldsymbol{u}}{\partial t}\right|^{t_{k}}-\left(\boldsymbol{u}^{k} \cdot \nabla\right) \boldsymbol{u}^{k}+v \nabla^{2} \boldsymbol{u}^{k}+\boldsymbol{g}\right)
$$

から導かれる，従って，粘性項を陰的に扱う場合には，新しい時刻の速度 $\tilde{\boldsymbol{u}}^{k+1}$ の発散が

$$
\nabla \cdot \tilde{\boldsymbol{u}}^{k+1}=\theta \Delta t \nabla \cdot\left[-v \nabla^{2} \boldsymbol{u}^{k}+v \nabla^{2} \tilde{\boldsymbol{u}}^{k+1}\right]
$$

となり， $\theta \neq 0$ のとき連続の式を満たさないことに注意が必要である．ただし，このとき発生する連続の式の残差 は, 次のステップの圧力ポアソン方程式で適切に考慮されるため, 時間発展に伴って蓄積するものではない. よっ て最終的な解に与える影響は軽微であると考えられる。 また, 流れが定常状態であれば $\nabla^{2} \boldsymbol{u}^{k}=\nabla^{2} \tilde{\boldsymbol{u}}^{k+1}$ であり, 解 析的には連続の式を満たすと言える.

\section{5. 数 值 検 証}

二次元 Taylor-Green 渦を検証問題として, 従来および提案の時間進行法によって得られる計算精度について比 較を行う。

\section{1 計算条件}

計算領域を図 1 に示すよう定義する。速度および圧力の解析解を以下に示す。

$$
\begin{aligned}
& u(x, y, t)=U \exp \left(-\frac{2 \pi^{2} t}{L^{2} / v}\right) \sin \left(\frac{\pi x}{L}\right) \cos \left(\frac{\pi y}{L}\right), \\
& v(x, y, t)=-U \exp \left(-\frac{2 \pi^{2} t}{L^{2} / v}\right) \cos \left(\frac{\pi x}{L}\right) \sin \left(\frac{\pi y}{L}\right), \\
& P(x, y, t)=\frac{\rho U^{2}}{4} \exp \left(-\frac{4 \pi^{2} t}{L^{2} / v}\right)\left(\cos \left(\frac{2 \pi x}{L}\right)+\cos \left(\frac{2 \pi y}{L}\right)\right) .
\end{aligned}
$$


式 (54) および式 (55) より速度の初期值を与え, 時間発展を計算する. 初期の粒子分布は正方格子状に等間隔に配 置する. 実際の計算では, 有次元パラメータの值として $L=1 \mathrm{~m}, \rho=1 \mathrm{~kg} / \mathrm{m}^{3}, v=10^{-3} \mathrm{~m}^{2} / \mathrm{s}$ を使用する。この ときレイノルズ数は $R e=L U / v=1000$ である.

提案手法では任意の粒子移動を扱うことができる。 そこで, Euler 法, Lagrange 法, ALE 法への適用を想定し, 粒子移動 $\Delta \boldsymbol{r}$ をそれぞれ以下のように定義する.

$$
\Delta \boldsymbol{r}^{k}= \begin{cases}\boldsymbol{0} & \text { (Eulerian) } \\ \Delta t\left(\frac{\boldsymbol{u}^{k}+\tilde{\boldsymbol{u}}^{k+1}}{2}\right) & \text { (Lagrangian) } \\ \Delta t\left(\frac{\boldsymbol{u}^{k}+\tilde{\boldsymbol{u}}^{k+1}}{2}\right)+\delta \boldsymbol{r}_{\text {shift }}^{k} & \text { (ALE) }\end{cases}
$$

$\mathrm{ALE}$ 法の場合には粒子分布の均一化のための補正項 $\delta r_{\text {shift }}^{k}$ を加えている. $\delta r_{\text {shift }}^{k}$ は以下のように与えるものとする.

$$
\begin{aligned}
& \delta \boldsymbol{r}_{\text {shift }, i}^{k}=-\frac{\alpha d \Delta x^{2}}{n^{0}} \sum_{j \neq i}\left[\frac{\boldsymbol{r}_{j}^{k}-\boldsymbol{r}_{i}^{k}}{\left\|\boldsymbol{r}_{j}^{k}-\boldsymbol{r}_{i}^{k}\right\|^{2}} w^{\prime}\left(\left\|\boldsymbol{r}_{j}^{k}-\boldsymbol{r}_{i}^{k}\right\|\right)\right] \\
& w^{\prime}(r)= \begin{cases}\frac{r_{\mathrm{e}}^{\prime}}{r}-1 & \left(r<r_{\mathrm{e}}^{\prime}\right) \\
0 & \left(r \geq r_{\mathrm{e}}^{\prime}\right)\end{cases}
\end{aligned}
$$

ここで $\alpha$ は均一化補正の強度を調整するための係数, $d$ は空間次元数, $n^{0}$ は $w^{\prime}$ を重み関数としたときの等間隔粒 子配置における粒子数密度を表す. 本研究では $\alpha=0.1, r_{\mathrm{e}}^{\prime}=2 \Delta x$ とした. また, 提案手法では粘性項を陰的に扱 うこともできるが, 本検証では従来手法にならって陽的に考慮するものとする.

空間離散化には 2 次および 4 次の LSMPS スキームを用いる. 影響半径 $r_{\mathrm{e}}$ は, 2 次のとき $r_{\mathrm{e}}=3.1 \Delta x, 4$ 次のと き $r_{\mathrm{e}}=4.1 \Delta x$ に設定し，スケーリング係数は $r_{\mathrm{s}}=r_{\mathrm{e}} / 2$ とする．速度および圧力の相対誤差ノルム $\left(L_{2}, L_{\infty}\right)$ をそれ ぞれ以下のように定義し,

$$
\begin{aligned}
& L_{2}^{u}=\sqrt{\frac{\sum_{i}\left\|\boldsymbol{u}_{\text {calc }, i}-\boldsymbol{u}_{\text {exact }, i}\right\|^{2}}{\sum_{i}\left\|\boldsymbol{u}_{\text {exact }, i}\right\|^{2}}} \\
& L_{\infty}^{u}=\frac{\max _{i}\left\|\boldsymbol{u}_{\text {calc }, i}-\boldsymbol{u}_{\text {exact }, i}\right\|}{\max _{i}\left\|\boldsymbol{u}_{\text {exact }, i}\right\|} \\
& L_{2}^{P}=\sqrt{\frac{\sum_{i}\left(P_{\text {calc }, i}-P_{\text {exact }, i}\right)^{2}}{\sum_{i}\left(P_{\text {exact }, i}\right)^{2}}} \\
& L_{\infty}^{P}=\frac{\max _{i}\left|P_{\text {calc }, i}-P_{\text {exact }, i}\right|}{\max _{i}\left|P_{\text {exact }, i}\right|}
\end{aligned}
$$

空間解像度 $(\Delta x)$ を変化させたときの䛊差ノルムの収束特性によって，それぞれの時間進行法の計算精度を評価する.

\section{$\mathbf{5 \cdot 2}$ 計算結果}

はじめに, Euler 法, Lagrange 法, ALE 法の比較のため, それぞれの場合に対し提案手法を適用したときの時刻 $t=0.3 \mathrm{~s}$ における計算結果を図 2 に示す。粒子分布は大きく異なるものの，おおよそ等しい圧力分布が得られてい ることがわかる. Lagrange 法と ALE 法を比較すると, Lagrange 法では粒子分布に大きな粗密差が発生している のに対して，ALE法では補正項の導入により均一な分布が得られている。 また， Lagrange 法の場合に更に時間発 展を進めると, 粒子分布の偏りに起因して数值不安定化が発生し, $t \sim 0.37 \mathrm{~s}$ で計算が破綻した. ALE 法ならびに Euler 法の場合には数值不安定化や数值解の不自然な挙動は確認されなかった. 


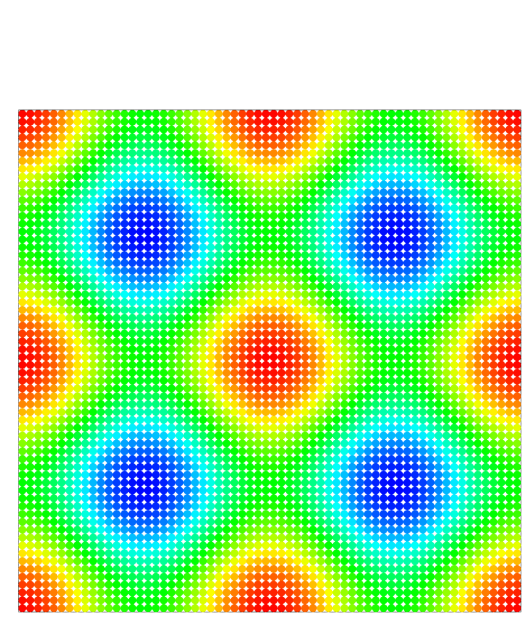

(a) Eulerian
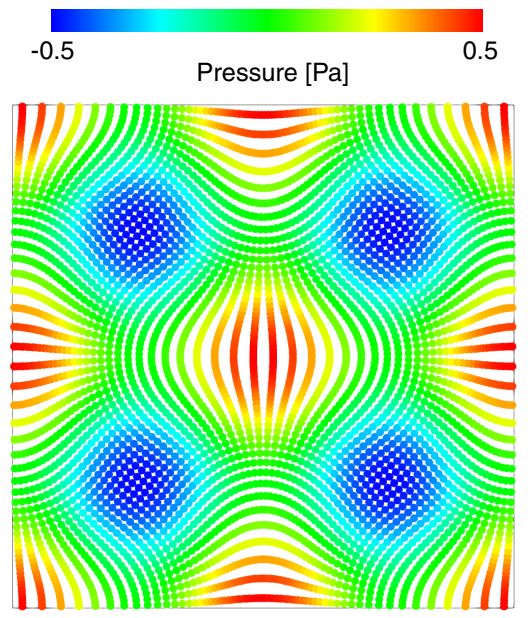

(b) Lagrangian

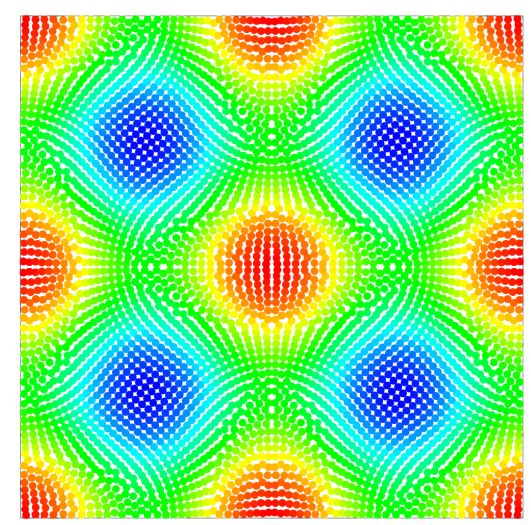

(c) ALE

Fig. 2 Comparison of the particle distribution and the pressure field obtained using the present time marching method at $t=0.3 \mathrm{~s}$. Agreement of the pressure is confirmed between different calculation framework. Highly-distorted particle distribution is found for the Lagrangian case. The number of particles is $64^{2}$.

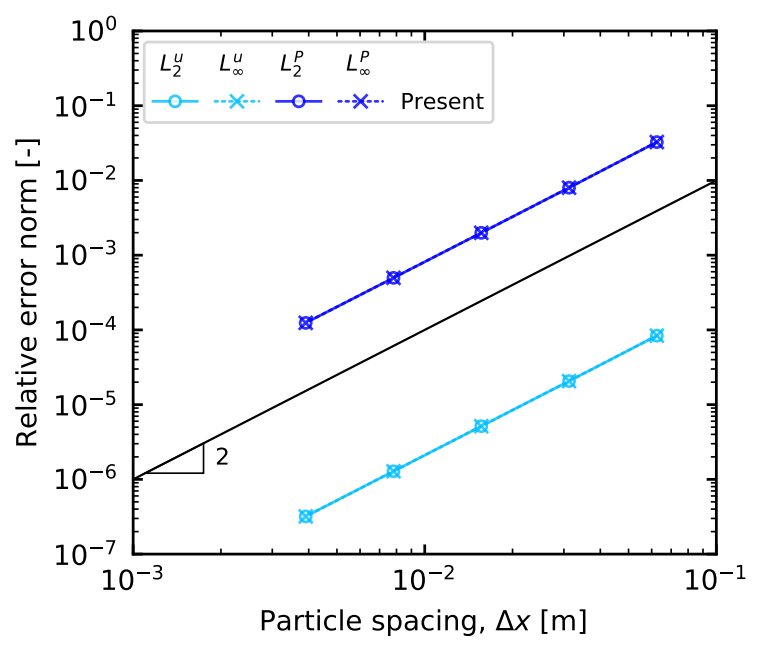

(a) 2nd-order LSMPS scheme

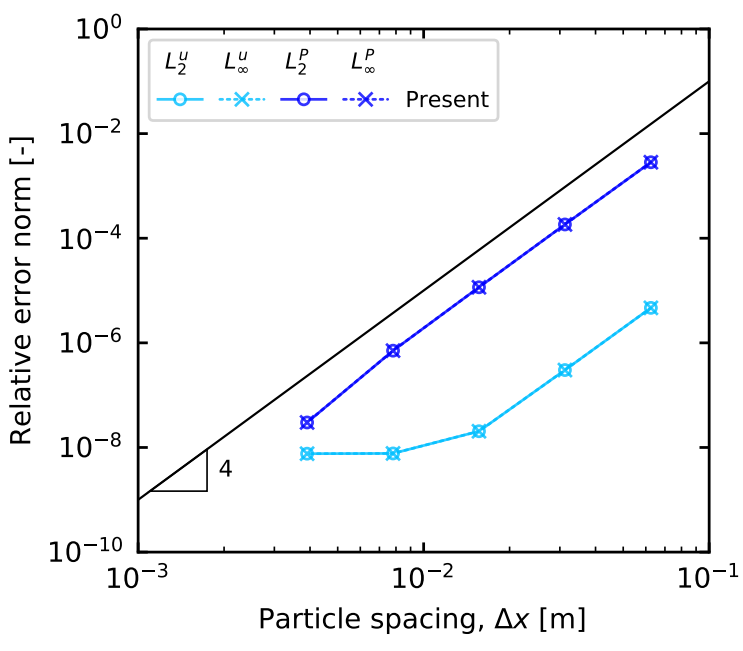

(b) 4th-order LSMPS scheme

Fig. 3 Convergence of the relative error norms obtained using present time marching method for a Eulerian model at $t=0.1 \mathrm{~s}$. The resulted convergence rates are coincident with the order of the spatial discretization scheme.

Euler 法の場合について, $\Delta t=2560^{-1} \mathrm{~s}$ に固定し $\Delta x$ を変化させたときの時刻 $t=0.1 \mathrm{~s}$ における誤差ノルムを図 3 に示す。空間離散化に 2 次の LSMPS スキームを用いたときには䛊差ノルムは 2 次のオーダーで減少し, 4 次の LSMPS スキームを用いたときにはおよそ4次のオーダーで減少しており，離散化スキームから期待される通りの 収束の傾きが得られていることがわかる。なお，4次のスキームを用いたときには，空間離散化に伴う $O\left(\Delta x^{4}\right)$ の 誤差が極めて小さくなるため, 部分段階法に含まれる $\mathscr{O}\left(\Delta t^{1}\right)$ の誤差が相対的に大きくなることで傾きが緩やかに なっている.

次に, Lagrange 法の場合について，従来の Koshizuka and Oka (1996)の時間進行法との比較を行つた。 上述と同 じ計算条件で取得した誤差ノルムの結果を図 4 に示す。図 4(a) は空間離散化に 2 次の LSMPS スキームを用いた とき，図4(b) は4 次の LSMPS スキームを用いたときの結果である. 従って，誤差ノルムは空間方向にそれぞれ 2 次および 4 次のオーダーで減少することが期待される。ところが，Koshizuka and Oka の方法では速度の誤差ノル ムの減少傾向は明らかにそれより低い. 2 次の LSMPS スキームを用いた場合の結果を見ると， $\Delta x$ の低下に伴って 傾きが緩やかになり，最終的にはほぼ横這いとなっている４４次の LSMPS スキームの場合に至っては，速度の誤 


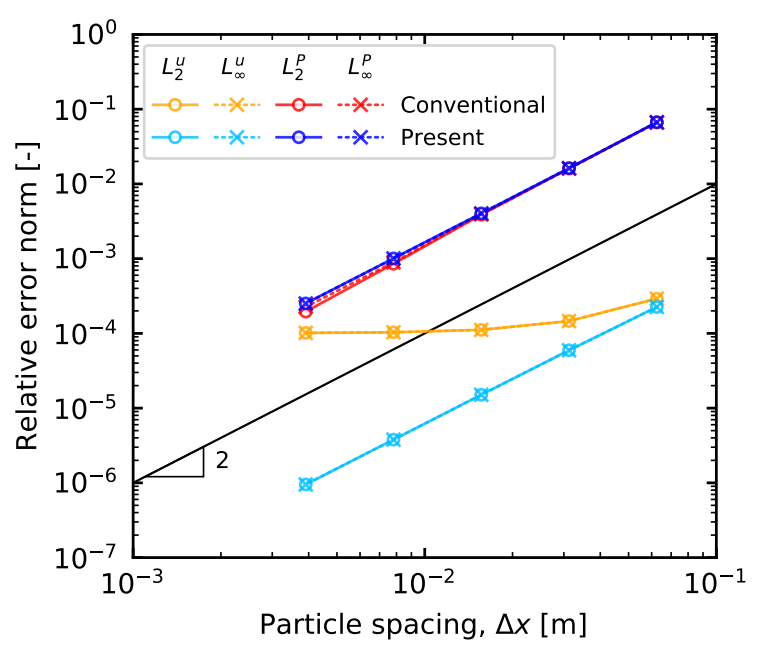

(a) 2nd-order LSMPS scheme

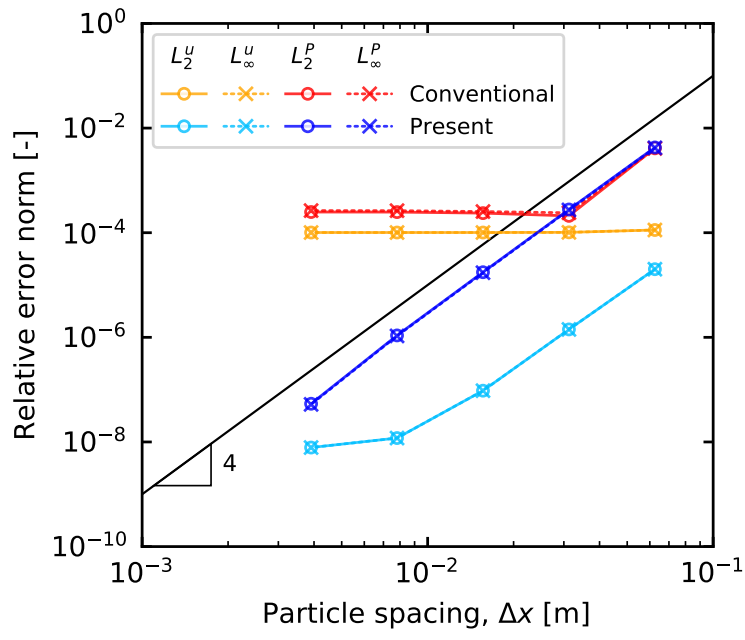

(b) 4th-order LSMPS scheme

Fig. 4 Comparison of the relative error norms between conventional and present time marching methods for a Lagrangian model at $t=0.1 \mathrm{~s}$. Spatial convergence is significantly improved by the present method.

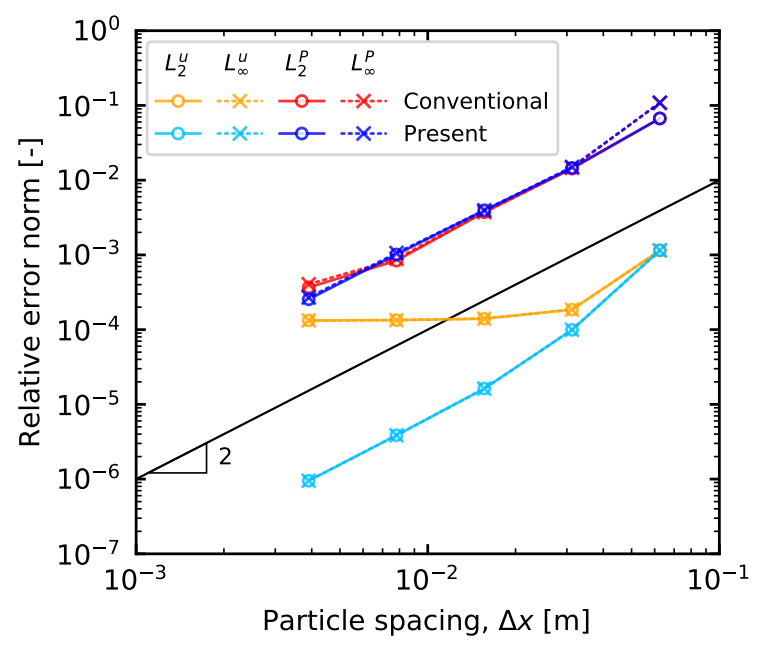

(a) 2nd-order LSMPS scheme

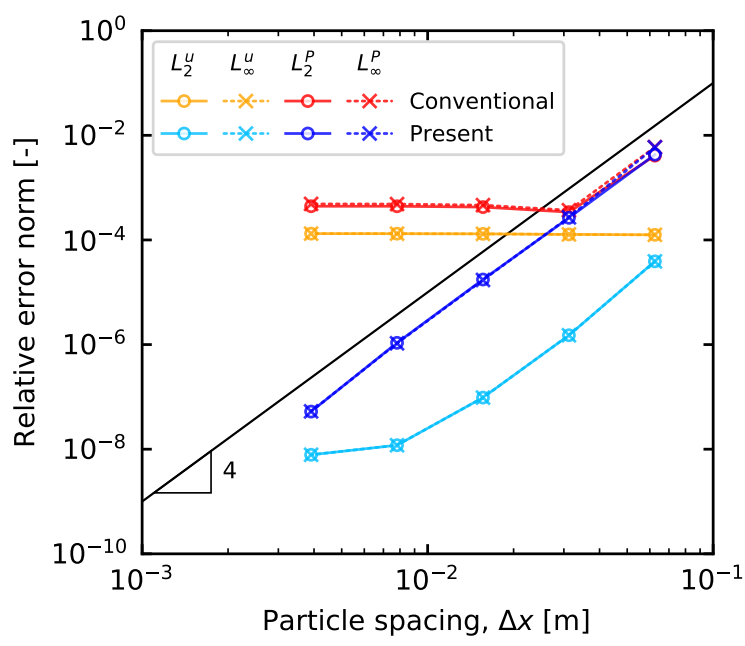

(b) 4th-order LSMPS scheme

Fig. 5 Comparison of the relative error norms between conventional and present time marching methods for an ALE model at $t=0.1 \mathrm{~s}$. Spatial convergence is significantly improved by the present method.

差ノルムの収束がほとんど確認できない.これは $\Delta t$ 依存の誤差が支配的であることを示している。結局，空間離 散化スキームを高次のものに置き換えても, 計算精度は向上しないということがわかる。一方, 提案手法では速 度・圧力共に誤差ノルムが概ね 2 次および 4 次のオーダーで減少しており，収束性が大きく改善されている４４次 の LSMPS スキームを用いた場合に, 誤差ノルムの傾きが次第に緩やかになっているのは, Euler 法の場合と同様 に, 相対的に $\Delta t$ 依存の誤差が大きくなってくるためである.このことから, 提案手法によって $\Delta t$ 依存の誤差の 大きさは 4 桁程度低減されたことがわかる.

最後に, ALE 法の場合について, 上述と同じ条件で従来の Matsunaga et al. (2020) の時間進行法との比較を行つ た。その結果を図 5 に示す. Matsunaga et al. の方法による結果は, Koshizuka and Oka の方法によるものとほぼ同 等であり, $\Delta t$ 依存の誤差が支配的となっていて空間方向の収束性は認められない. 提案手法では, Lagrange 法の 場合と同様に，速度・圧力共に誤差ノルムが概ね 2 次および 4 次のオーダーで減少することが確認できる.よつ て，均一化補正を加えた ALE 法に対しても提案手法は有効であることが示されている. 


\section{6. 結 言}

本研究は，粒子法の計算精度の向上を目的として，新しい時間進行法を提案するものである. 従来手法において 収束を阻害する要因になっていると考えられる時間ステップ依存の誤差に着目し，その発生原因を指摘した。提案 の時間進行法は，従来と同じく時間 1 次精度の部分段階法に基づくものである.ALE 法を対象としており，任意 の粒子移動を扱うことができる. 従来手法と大きく異なる点は, 対流項を陽的に計算式に組み込み, 粒子移動によ る速度の変化を Taylor 展開近似によって定式化することである。それによって，対流項に現れる誤差項ならびに 仮速度の定義位置のずれの問題を解消し，これらに伴う時間ステップ依存の誤差の発生を根本的に回避する。数 值検証として, 二次元 Taylor-Green 渦に対して与えられる誤差ノルムの収束性を評価し, 従来手法との比較を行つ た。その結果，時間ステップ依存の誤差が大きく低減されたことで，従来手法では認められなかった空間 2 次お よび 4 次の収束性が得られた。 よって, 提案の時間進行法によって計算精度が大きく向上されることが示された。

\section{References}

Cummins, S.J. and Rudman, M., An SPH projection method, Journal of Computational Physics, Vol.152 (1999), pp.584607.

Duan, G., Koshizuka, S., Yamaji, A., Chen, B., Li, X. and Tamai, T., An accurate and stable multiphase moving particle semi - implicit method based on a corrective matrix for all particle interaction models, International Journal for Numerical Methods in Engineering, Vol.115 (2018), pp.1287-1314.

Gotoh, H. and Khayyer, A., Current achievements and future perspectives for projection-based particle methods with applications in ocean engineering. Journal of Ocean Engineering and Marine Energy, Vol.2 (2016), pp.251-278.

Hu, F., Matsunaga, T., Tamai, T. and Koshizuka, S., An ALE particle method using upwind interpolation, Computers \& Fluids, Vol.145 (2017), pp.21-36.

Khayyer, A., Gotoh, H. and Shimizu, Y., Comparative study on accuracy and conservation properties of two particle regularization schemes and proposal of an optimized particle shifting scheme in ISPH context, Journal of Computational Physics, Vol.332 (2017), pp.236-256.

Koshizuka, S. and Oka, Y., Moving-particle semi-implicit method for fragmentation of incompressible fluid, Nuclear Science and Engineering, Vol.123 (1996), pp.421-434.

Koshizuka, S., Shibata, K., Kondo, M. and Matsunaga, T., Moving particle semi-implicit method: A meshfree particle method for fluid dynamics (2018), Academic Press.

Matsunaga, T., Södersten, A., Shibata, K. and Koshizuka, S., Improved treatment of wall boundary conditions for a particle method with consistent spatial discretization, Computer Methods in Applied Mechanics and Engineering, Vol.358 (2020), 112624.

Monaghan, J.J., Smoothed particle hydrodynamics, Annual Review of Astronomy and Astrophysics, Vol.30 (1992), pp.543-574.

Oger, G., Marrone, S., Le Touzé, D. and de Leffe, M., SPH accuracy improvement through the combination of a quasiLagrangian shifting transport velocity and consistent ALE formalisms, Journal of Computational Physics, Vol.313 (2016), pp.76-98.

Tamai, T. and Koshizuka, S., Least squares moving particle semi-implicit method, Computational Particle Mechanics, Vol.1, No.3 (2014), pp.277-305.

Violeau, D. and Rogers, B.D., Smoothed particle hydrodynamics (SPH) for free-surface flows: past, present and future, Journal of Hydraulic Research, Vol.54 (2016), pp.1-26.

Wang, Z., Duan, G., Matsunaga, T. and Sugiyama, T., Consistent Robin boundary enforcement of particle method for heat transfer problem with arbitrary geometry, International Journal of Heat and Mass Transfer, Vol.157 (2020), 119919.

$\mathrm{Xu}$, R., Stansby, P. and Laurence, D., Accuracy and stability in incompressible SPH (ISPH) based on the projection method and a new approach, Journal of Computational Physics, Vol.228 (2009), pp.6703-6725. 